\title{
CELLULAR NORMAL MODES OF ALLUVIUM RESPONSE
}

\author{
W. R. Stephenson*
}

\section{ABSTRACT}

The concept of directed resonance is put forward, and its occurrence in New Zealand and overseas is documented. It is observed mainly on watersaturated recent alluvium. Analytical procedures for detecting directed resonance effects in earthquake records are outlined. The resonance parameters are shown to be independent of the particular earthquake, and highly dependent on local boundaries of the alluvium.

Cellular mode resonant response is suggested to be the mechanism generating directed resonance at individual sites, and is shown to occur for scale models of alluvial deposits.

Experimental apparatus which would be needed to unequivocally confirm the earthquake excitation of cellular resonant modes is described in an appendix.

\section{INTRODUCTION}

It is now widely recognised that the earthquake-induced ground motion at a site can be greatly influenced by local geomechanical properties. In particular, deep $(>10 \mathrm{~m}$ ) soft alluvial deposits have often been observed to magnify the effects on structures.

In the most commonly adopted model used to account for the influence of local geology, it is assumed that the site can be analysed as infinite horizontal layers of flexible material (1). Multiple reflections at the boundaries of these layers then give resonance effects which cause substantial differences between assumed base-rock motions and computed surface motions.

When the site has steeply sloping boundaries between layers, or steeply sloping soil-rock boundaries, a two-dimensional finite-element model is sometimes adopted (2). For both these models the computed surface motions have the same direction as the causative base-rock motions. However, as a consequence of the present investigations this result is aisputed.

To get an exact picture of the processes responsible for the site-dependence of ground motion, one must solve the wave equation in the medium, for the relevant initial conditions and boundary conditions. Two broad approaches to finding analytical solutions have been used for a long time, and the usefulness of each depends on the particular geometry involved.

For situations involving dimensions much larger than a wavelength, or involving gradual changes of materials such that boundaries are remote or hard to identify. wave propagation approaches are appropriate. on the other hand when a sharply bounded

* Physics and Engineering Laboratory,

D.S.I.R., Lower Hutt, New Zealand. medium is excited generally (as opposed to locally), normal mode approaches are most fruitful.

Thus the propagation of earthquake waves from the source, through various layers of the earth to a building, is thought of as a wave propagation problem, while the response of the building is often considered to be the sum of responses of normal modes.

In what follows, the microzone problem is considered as the effects of the nearly synchronous arrival of seismic input on a local pocket of alluvium sharply bounded by hard rock. Thus we are considering the site-dependence of ground motion to be caused by the excitation of physically-real easily-visualised normal modes.

\section{SITE OBSERVATIONS}

\subsection{General}

During the past two years field measurements have been made by the author on selected alluvium, and on base-rock, in the wellington area of New zealand, in order to test the adequacy of existing models of local soil deposits.

The usual analyses, in which the response spectra of measured surface motions are compared with the response spectra of computed surface motions, have been applied to the measurements but have been found to be deficient. This arises because response spectra tend to conceal subsidiary features of the ground motion which become the dominant features during another earthquake having a base-rock motion of different spectral composition.

However, useful information can be obtained from examination of the power spectra of earthquakes recorded at individual sites. The earthquakes were recorded by the techniques given in Appendix 1. 


\subsection{Directed Resonance}

An examination of the power spectra of earthquake motions recorded on flat ground as functions of azimuth divides the earthquake motions sharply into two classes. In the case of motions measured on weak, water-saturated alluvium a "directed resonance" is invariably observed, but in no other recorded case is there seen any dramatic directional feature.

"Directed resonance" is characterised by the type of power spectrum shown in Fig. I. where there are two distinct spectral components, namely a relatively uniform, isotropic noise-like background, and a sharp resonance, which is highly directional. These properties have led to the author adopting the name "directed resonance" to describe this significant phenomenon.

The field work has provided many instances of directed resonances. For example, the earthquake of January 6 th, 1973, which was sufficiently intense to start most of the strong motion recorders in the Wellington area, provided the power spectra shown in Fig. 1 and Figs. $3-12$. These results are displayed to show specific site resoriance parameters (and hence possible future resonant frequencies) as well as to demonstrate the occurrence of directed resonance on soft soil and on a rocky ridge (a "structural" directed resonance). They also show the non-occurrence of resonance on flat bed-rock.

Two significant observations were made during the same earthquake. As Figs. 1, 3 and 4 show, this amounted to the observation of directed resonant motion at the close pair of alluvial sites (Physics and Engineering Lab. and Elizabeth St.) with the resonant motion coherent between the two sites but differing in direction for each. In addition Table I shows that, at the Physics and Engineering Lab. site which is near the rock boundary, resonant motion for a range of earthquakes of different intensities and epicentres had nearly constant frequency and direction. These observations are consistent with a behaviour of alluvium which the author has named "cellular-mode response".

The existence of resonant behaviour along cne axis but not at right angles to it, may be seen in the ground velocity record of Fig. 2. Here, the instrument areas were deliberately aligned along the suspected resonance direction so that the phenomenon would be observed without the need for complicated analysis. The $50^{\circ}$ component shows resonant behaviour.

\subsection{Comment}

Why is it that directed resonances have not been reported before now? Identifying them depends on finding a resonant peak along one direction, and establishing its absence at right angles to that airection. However, the choice of two arbitrary orthogonal directions is unlikely to be appropriate to showing up directed resonance. mainly to saturated alluvium this further reduces the chance of accidental discovery.

Again a response spectrum analysis is less likely to show up resonant peaks than a power spectrum analysis. Hence looking at response spectra along instrument sensing axes, for arbitrary records, is unlikely to show up directed resonance.

It is the author's experience that, using the analytical procedures of section 3.2 of this paper, directed resonance is invariably detected on recent saturated alluvium under earthquake excitation. However, directed resonances need not be restricted to alluvium response. The simplest counter-example is of a building whose longitudinal and transverse mode frequencies are different, when each resonance will be shown only along the appropriate building axis.

Because the observation of directed resonance is so much at variance with deductions made from present models it is desirable that at least a physical picture, and preferably a mathematical model, be found for the special case of water-saturated alluvium excited by earthquake accelerations.

\section{CELLULAR MODE RESPONSE}

\section{I Development of Analytical Method}

It is implicit in the infinite layer model (I) that the motion of an alluvial deposit in any direction can be described in terms of rock motion in the same direction and a soil impulse function.

This assumption of independence of components was shown to be wrong in the Hutt Valley, to the extent that in one direction soil motion was occasionally found to precede the same component of rock motion. This soil motion had evidently arisen from the unmeasured component of rock motion.

Granted that both horizontal components of rock motion contribute to each horizontal component of alluvium motion, one can try to extend the linear theory implicit in the idea of impulse response; or, alternatively, one can look for a totaliy different physical picture to use in modelling. The former could consider vector, rather than scalar, time sequences for inputs and outputs, and a matrix, rather than scalar, time sequence for the impulse function.

The matrix impulse function theory has already proved inadequate and a search for a new model has been carried out, based on the concept of "directed resonances". It has led to a physical picture which the author has named "cellular mode resonance".

According to this picture the surface of an alluvial deposit can be divided into two-dimensional cells, each of which has unique resonant properties. In each cell the alluvium moves coherently in a resonant fashion, its resonant frequency being different from that of its neighbour. The cell boundaries are determined exclusively by the physical boundaries of the alluvium so that the size of a cell is related to the large scale geological setting.

Although the above attempts to supplant 
the infinite layer model and two-dimensional finite element model it has the status of a physical picture rather than a mathematical model. However, the concept of large cells with coherent motion represents a useful advance, and may eventually give rise to a complete mathematical model.

\subsection{Analysis}

The power spectrum of a variable $A(t)$ is defined as ${ }_{\infty}$

$$
P(E)=\int_{-\infty}^{\infty} \int_{-\infty}^{\infty} A(\tau) A(t+\tau) e^{-2 \pi i f t} d t d \tau
$$

where $t, \tau$ and $f$ have their usual significance. If the variable $A$ has components $A_{N}$ and $A_{E}$ in directions north and east, we can express its value in the direction $\theta$ clockwise from north as

$$
A_{\theta}=A_{E} \sin \theta+A_{N} \cos \theta,
$$

and its power spectrum as

$$
\begin{aligned}
& P_{\theta}(f)=\int_{-\infty}^{\infty} \int_{-\infty}^{\infty}\left(A_{E}(\tau) \sin \theta+A_{N}(\tau) \cos \theta\right) \times \\
& \left(A_{E}(t+\tau) \sin \theta+A_{N}(t+\tau) \cos \theta\right) e^{-2 \pi i f t} d t d \tau \\
& =\sin ^{2} \theta \int_{-\infty}^{\infty} \int_{-\infty}^{\infty} A_{E}(\tau) A_{E}(t+\tau) e^{-2 \pi i f t} d t d \tau \\
& +\sin \theta \cos \theta \int_{-\infty}^{\infty} \int_{-\infty}^{\infty} A_{E}(\tau) A_{N}(t+\tau) e^{-2 \pi i f t} d t d \tau \\
& +\sin \theta \cos \theta \int_{-\infty}^{\infty} \int_{-\infty}^{\infty} A_{E}(t+\tau) A_{N}(\tau) e^{-2 \pi i f t} d t d \tau \\
& +\cos ^{2} \theta \int_{-\infty}^{\infty} \int_{-\infty}^{\infty} A_{N}(\tau) A_{N}(t+\tau) e^{-2 \pi i f t} d t d \tau
\end{aligned}
$$

As the $A_{N}$ and $A_{E}$ are real, the middle two terms of equation (3) are complex conjugates and we may use

$$
P(f)=2 \int_{0}^{\infty} \int_{0}^{\infty} A(\tau) A(t+\tau) \cos 2 \pi f t d t d \tau
$$

and

$$
P_{\theta}(f)=P_{N} \cos ^{2} \theta+P_{E} \sin ^{2} \theta+2 P_{x} \sin \theta \cos \theta
$$

$P_{N}$ is the north power, obtained by substituting $A_{N}$ for $A$ in equation (4), and similarly $\mathrm{P}_{\mathrm{E}}$ is the east power. $\mathrm{P}_{\mathrm{X}}$ is the crosscomponent power obtained as

$$
P_{X}=2 \int_{0}^{\infty} \int_{0} P_{N}(\tau) P_{E}(t+\tau) \cos 2 \pi f t d t d \tau
$$

The power in the direction $\theta$ may be reexpressed as

$$
\begin{aligned}
& P_{\theta}(f)=\frac{P_{N}+P_{E}}{2}+\left[P_{X}^{2}+\left(\frac{P_{N}-P_{E}}{2}\right)^{2}\right]^{\frac{1}{2}} x \\
& \cos \left(2 \theta-\tan ^{-1} \frac{2 P_{X}}{P_{N}-P_{E}}\right)
\end{aligned}
$$

From equation (5) we see that the spectrum for any azimuth may be calculated from the three fundamental spectra $P_{E}, P_{N}$

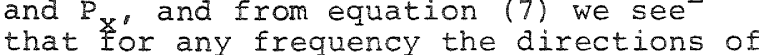

maximum and minimum spectral ordinate may easily be calculated.

Thus to perform a directional resonance analysis, only the power spectrum of $A_{N}$ and $A_{F}$ and the cross power spectrum between them need be calculated.

At this stage the bulk of the computing has been done, and resonance directions and spectra for specific directions are easily found, even on a desk calculator.

The usual rules for spectral analysis apply, and in the spectra shown here the results have been band limited by applying Gaussian time windows to the lag correlograms. The apertures have been chosen to be functions of the frequency, so that at any frequency the bandwidth is adequate to give the full response of a resonator with a $Q$ of less than 10.

\subsection{Comment}

The mechanical properties of watersaturated alluvium and of liquids have many similarities. Both are almost incompressible and the low shear modulus exhibited by the alluvium is replaced by a zero shear modulus in the case of a liquid. It is the combination of being easy to shear but hard to compress that allows simple physical reasoning to be applied in predicting the behaviour of bodies of saturated alluvium in rigid rocky basins. In addition to gravity we expect to see shear elasticity acting as a restoring force. Thus there will be two sorts of waves. Considering the resonances associated with these waves we can expect seiche-like resonances exhibiting nearly vertical motion, and cellular mode resonances. The latter will consist of harmonic variations of a field of horizontal vectors which have no divergence and hence follow lines of possible liquid flow.

Such cellular-mode resonances can be readily observed by exciting a shallow tray of a gelatine-and-water jelly in a horizontal plane. Many useful insights can be obtained by such model studies, and they lead directly to ideas of directed resonance, and pairs of directed resonances due to mode superposition such as those of Figures 8 and 9. They also show how cell boundaries and resonance directions are determined almost solely by boundary geometry. Figure 19 is a time exposure showing displacements due to a cellular mode resonance in such a model. The model base was subjected to a sinusoidal translation directed along the irregular boundary, at the mode natural period. It is a clear example of the flowlike character of such modes. The torsion evident in the model will also occur in nature, but it is to be noted that the motion at any point when considered over the area of an appropriately scaled building, is linear and sinusoidal. That is, any point on the model shows a directed resonance. For the model illustrated the variation of amplitude is expected to be a quarter sine wave vertically and a first order Bessel function of the first kind $\left(J_{1}(r)\right)$ radially.

Cellular mode response is initiated by a translation of a whole boundary system and the elastic medium it contains. Micro- 
tremors on the other hand are known to result from local vibration sources, to persist only to shallow depths, and to consist mainly of travelling surface waves. When techniques designed to show directed resonance are applied to microtremor records from a site known to have a directed resonance under earthquake excitation, no such directed resonant response is noted. It is concluded that microtremor analysis is not a suitable tool to investigate cellular mode response.

It is very advantageous to have the ground and structural periods for any given case different by at least a factor or two. However, it will be noticed that the frequencies reported in this paper are characteristic of many New zealand buildings. It is expected that during strong shaking both ground and structural periods will increase, the ground period increase being greater. A coincidence of ground and structural period can be avoided by modifying the structural form. For a given structure, shear walls will result in a comparatively short structural period, or for buildings up to 10 storeys a base isolation system (4) will result in a lengthening of the structural period and lower loads. In this latter case, one would have to avoid the ground period "catching up" with the structural pericds for grossly nonlinear soils.

\section{OVERSEAS RECORDS}

Figures 13 to 18 shor the occurrence of directed resonance in several overseas earthquakes. Of particular interest are the Leukas and Niigata analyses, because of the intensities at the measuring sites. Leukas shows the change in resonant character clue to a change in intensity by allowing comparison of the $0.54 \mathrm{~g}$ main shock with the $0.1 \mathrm{~g}$ aftershock, and the Niigata analysis shows a directed resonance occurring in the presence of vibrations severe enough to induce liquefaction. Cellular mode response involving a progressive loss of shear stiffress, but not compressional stiffness, as liquefaction occurs, can account for these results which are unexpected on multiple reflection or shear beam theory, as explained in the section on cellular mode resonance.

\section{CONCLUSIONS}

Many mechanisms have been proposed to account for microzone damage variations, and these have indicated increases in accelerations for particular geological settings combined with particular earthquakes.

However, the most startling contrasts in seismic damage have always been seen between structures built on saturated recent alluvium and those built on nearby competent ground. Clearly, the most significant advance will come from explaining the salient features of the response of such alluvium, and how it is that for particular earthquakes damage is avoided in some structures.

Cellular mode response is particular to saturated recent alluvium; and its importance during a particular earthquake depends on how the earthquake energy is distributed with respect to frequency. Its importance to a particular structure depends on the closeness of structural mode frequencies to the ground resonant freouency.

The discovery of directed resonance, and its agreement with the concept of cellular modes suggests that it may be possible to characterise local regions as resonators and to avoid inadvertent coincidences of ground and structural natural periods, or make provision for increased forces.

The crucial points are that the most dramatic damage enhancements have been seen on soft water-saturated alluvium, that cellular mode responses seem to be associated with the same alluvium, and that cellularmode responses show promise of repeatable behaviour which is relatively amplitude independent.

\section{REFERENCES}

(1) Idriss, I. M. and Seed, H. B., "Seismic Response of Horizontal Soil Layers". J. Soil Mech. Fdns. Div. Am. Soc. Civ. Engrs. 94: SM4 p 1003 (1968).

(2) Idriss, I. M. "Finite Element Analysis for the Seismic Response of Earth Banks". J. Soil Mech. Fdns. Div. Am. Soc. Civ. Engrs. 94 : SM5 p 617 (1968).

(3) Stephenson, W. R. "Microzoning for Design" in "Structural Design for Earthquakes", University of Auckland, New Zealand, August, 1972.

(4) Skinner, R. I., Beck, J. L. and Bycroft, G. N. "A Practical System for Isolating Structures from Earthquake Attack". Intl. J. of Eq. Eng. and Struct. Dyn. 1975 (In print).

\section{APPENDIX 1}

\section{An Experimental System}

In view of the importance of demonstrating and predicting cellular mode resonance effects, a way of rapidly obtaining information about an area by frequently operating a small network of recorders has been devised. This does not eliminate a need for supplementary strong-motion data which is the only valid basis for investigating amplitude effects. By using small earthquakes we can rapidly gain information on the simpler linear case, which must be included in the general case.

Ground motion measurements made during small earthquakes should be synchronous at all sites in order to interrelate them correctly, and they should have sufficient resolution $(0.1 \%)$ and bandwidth $(0-25 \mathrm{~Hz})$ to define accurately a wide range of amplitudes and frequencies. This calls for 500 bits per second per component, which immediately rules out continuous recording for any long interval of time. One must choose between commanded field recording, and continuous field monitoring with triggered base recording. With commanded field recording data degradation due to transmission can be avoided, and the power requirements can be concentrated at a fixed base station. During standby the field stations need only monitor the command channel, and this requires very little power. 
The system is as follows. Vertical ground velocity is sensed and processed by a unit installed in a tunnel in hard rock so that the arrival of p-waves can be established. Motion of this hard bed-rock due to non-earthquake events has spectral and duration properties which are different from earthquakes, and by clipping, filtering and integrating, such a signal can be suppressed, and any earthquake which results in a soil signal greater than ambient vibrations may be detected. Such events occur in Wellington about once a week.

The command signal derived from the triggering site is transmitted by radio to the field stations together with timing pulses, and also a reference tone which may be used to establish the recording speeds of the field tape recorders.

Each field station consists of a pair of horizontally sensing accelerometers and amplifiers which drive voltage-tofrequency converters. On command, the resultant two ground motion tones, the reference tone and the timing pulses are recorded on a tape recorder.

Replay of a magnetic tape record is performed through three filters and frequency-to-voltage converters. This results in voltages corresponding to the two components of ground acceleration and to the error between record and replay tape speeds. A digital computer has been programmed to sample these voltages as each time mark occurs, and this makes the ground motion data readily available in a convenient form. The same computer, or a more easily accessible one, can then be used to perform a directional resonance analysis of the ground motion.

\section{TAELE 1}

Date, resonance parameters and approximate peak acceleration, for five earthquakes recorded at the Physics and Engineering Laboratory. (Saturated alluvium near a rock boundary.)

\begin{tabular}{|c|c|c|c|}
\hline DATE & RESONANCE DIRECTION & RESONANT FREQUENCY & ACCELERATION \\
\hline $1 / 11 / 68$ & $51^{\circ}$ & $2.4 \mathrm{~Hz}$ & $0.15 \mathrm{~g}$ \\
\hline $24 / 1 / 71$ & $40^{\circ}$ & $2.1 \mathrm{~Hz}$ & $0.0002 \mathrm{~g}$ \\
\hline $6 / 1 / 73$ & $65^{\circ}$ & $2.6 \mathrm{~Hz}$ & $0.03 \mathrm{~g}$ \\
\hline $26 / 3 / 73$ & $59^{\circ}$ & $2.9 \mathrm{~Hz}$ & $0.03 \mathrm{~g}$ \\
\hline $28 / 12 / 73$ & $50^{\circ}$ & $3.2 \mathrm{~Hz}$ & $0.0002 \mathrm{~g}$ \\
\hline
\end{tabular}



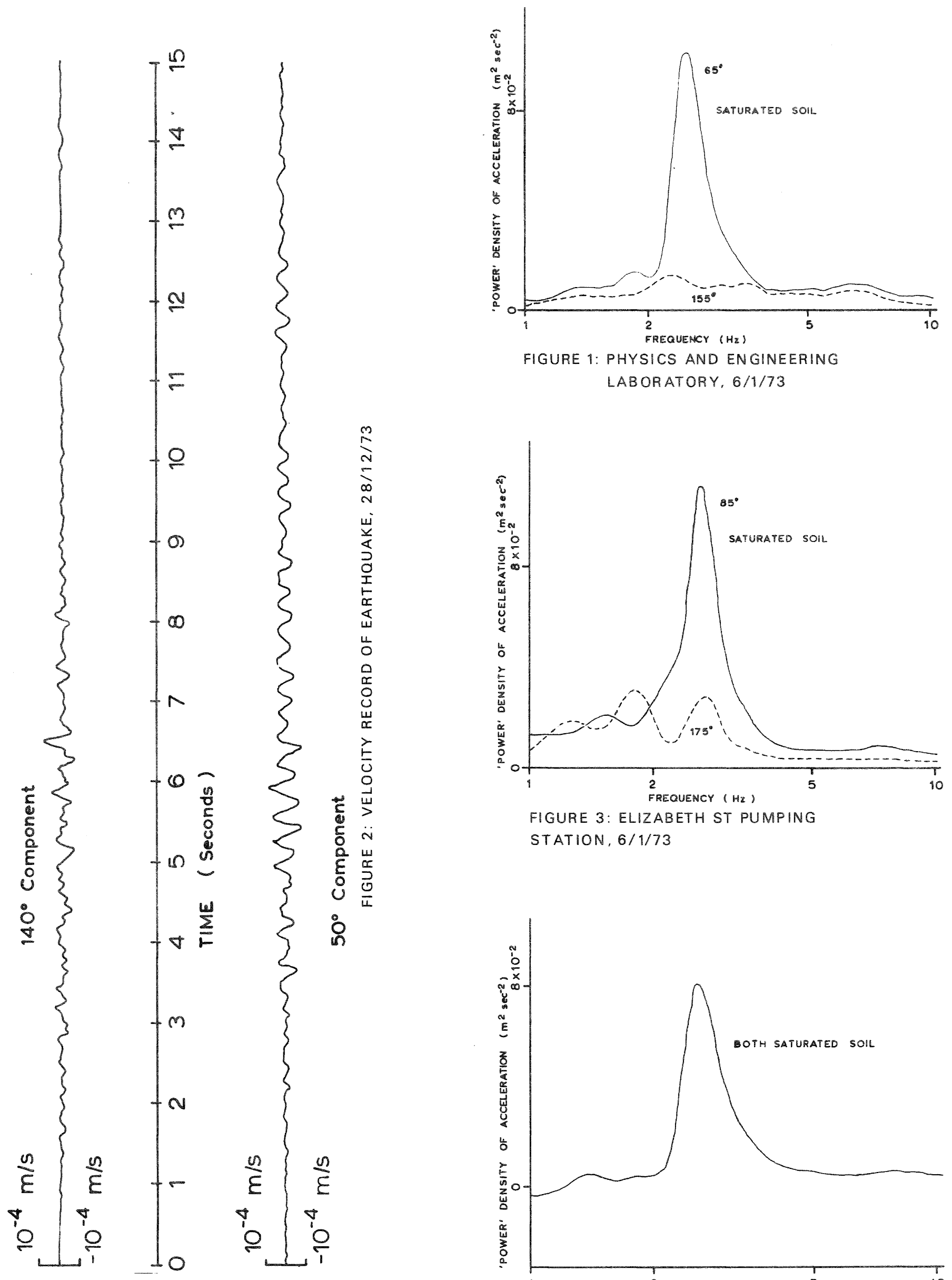

FIGURE 1: PHYSICS AND ENGINEERING LABORATORY, 6/1/73

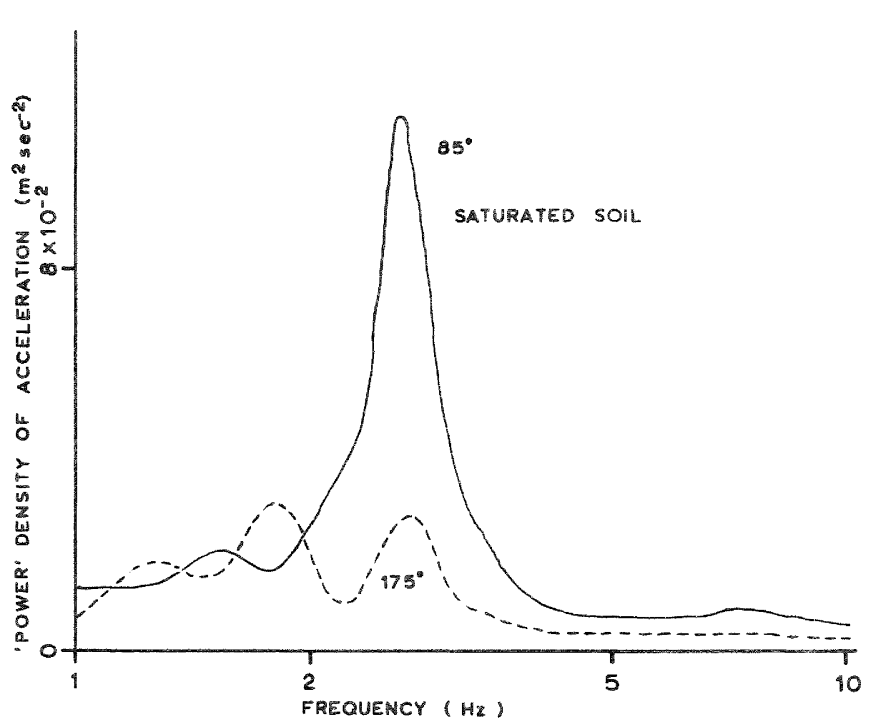

FIGURE 3: ELIZABETH ST PUMPING STATION, 6/1/73

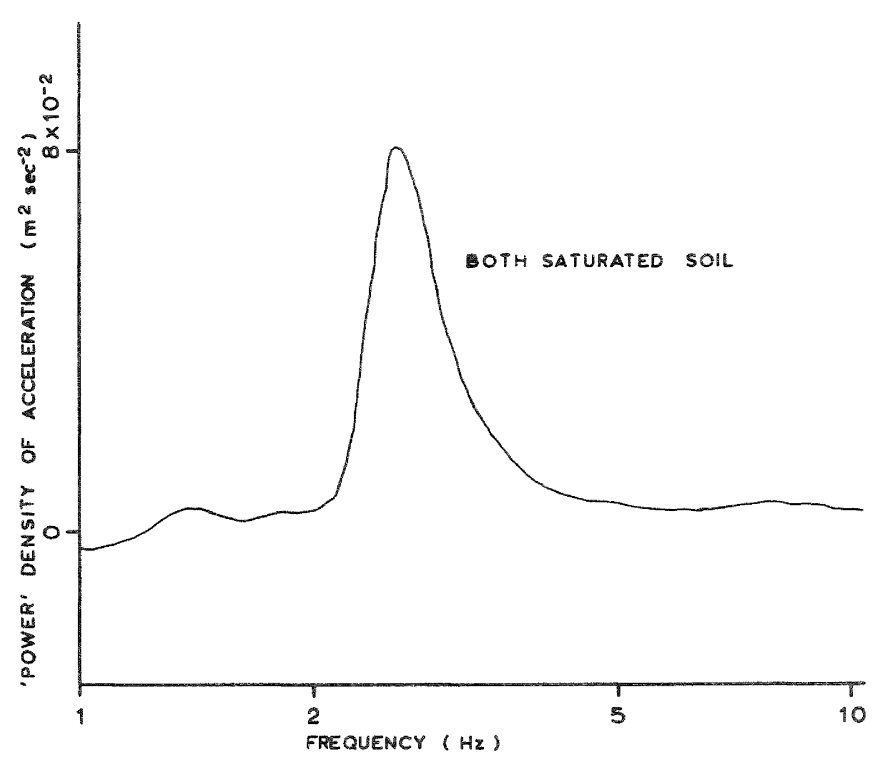
FIGURE 4: CROSS-SPECTRUM OF PEL AT $65^{\circ}$ AND ELIZABETH ST AT $85^{\circ}, 6 / 1 / 73$ 


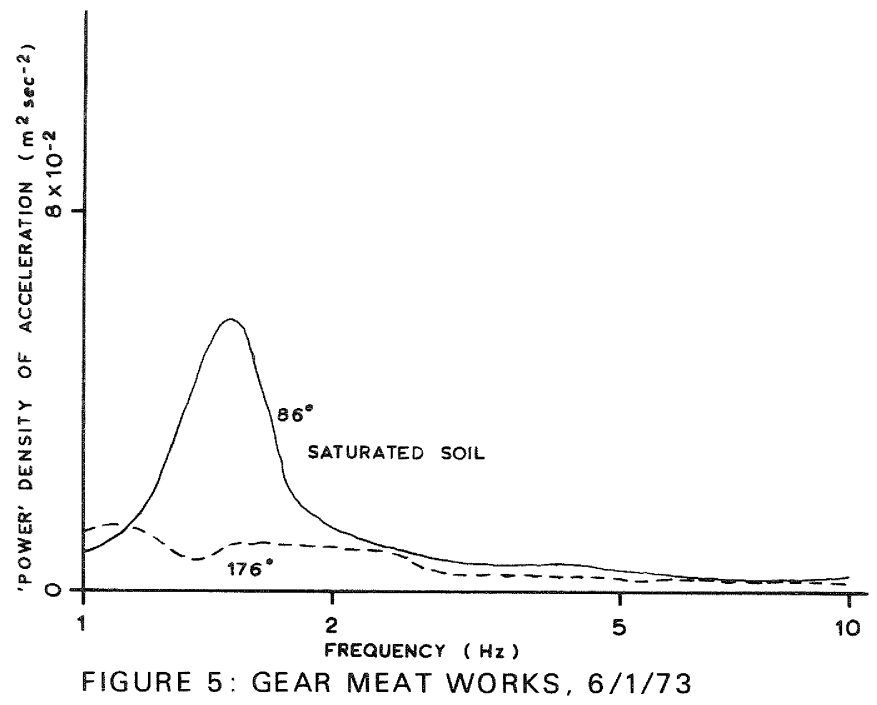

FIGURE 5: GEAR MEAT WORKS, 6/1/73

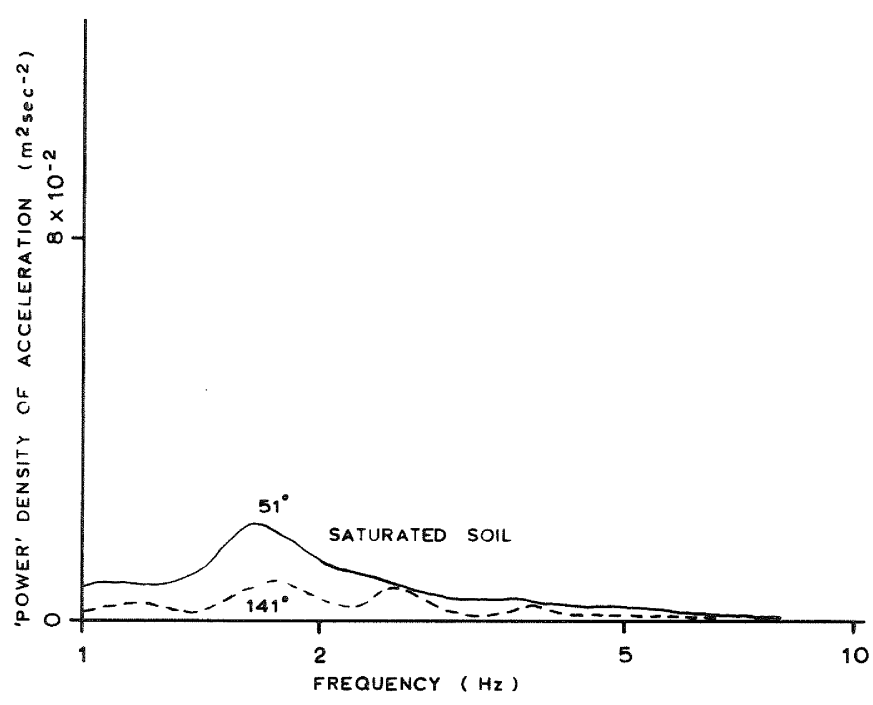

FIGURE 7: WELLINGTON AIRPORT, $6 / 1 / 73$

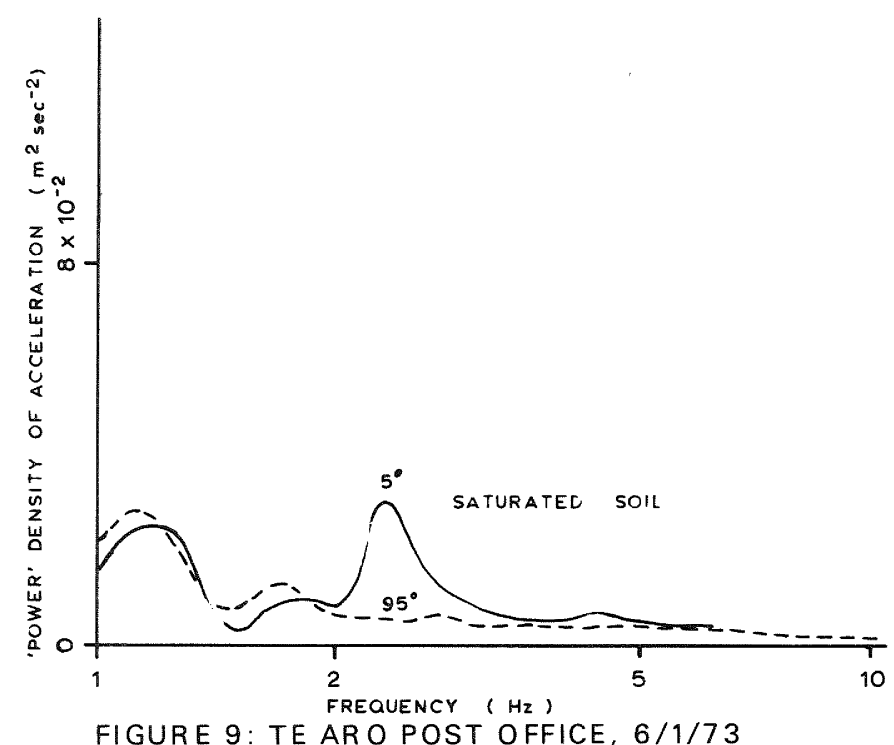

FIGURE 9: TE ARO POST OFFICE, $6 / 1 / 73$

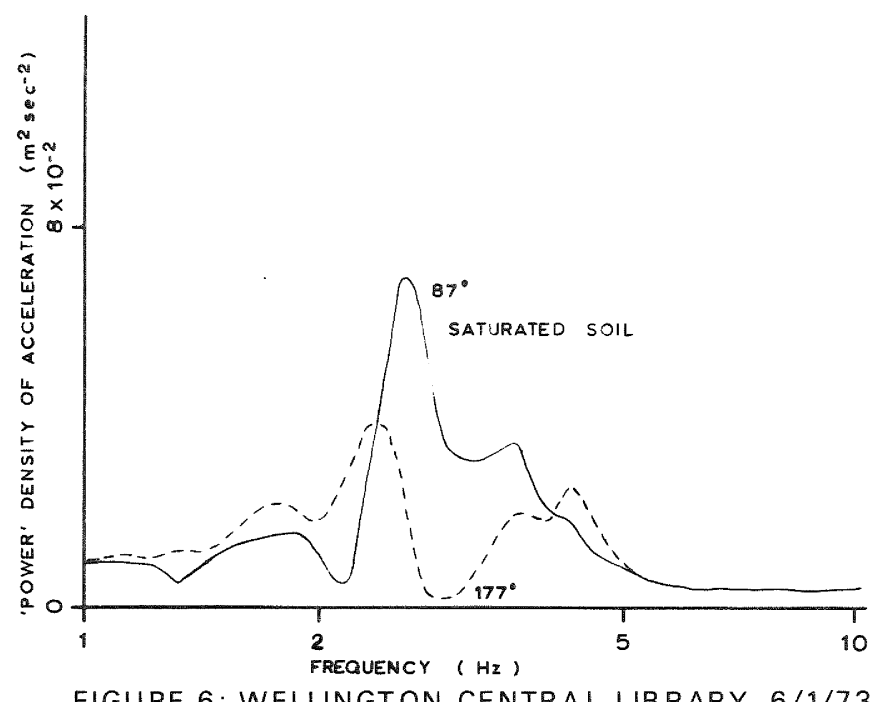

FIGURE 6: WELLINGTON CENTRAL LIBRARY, 6/1/73

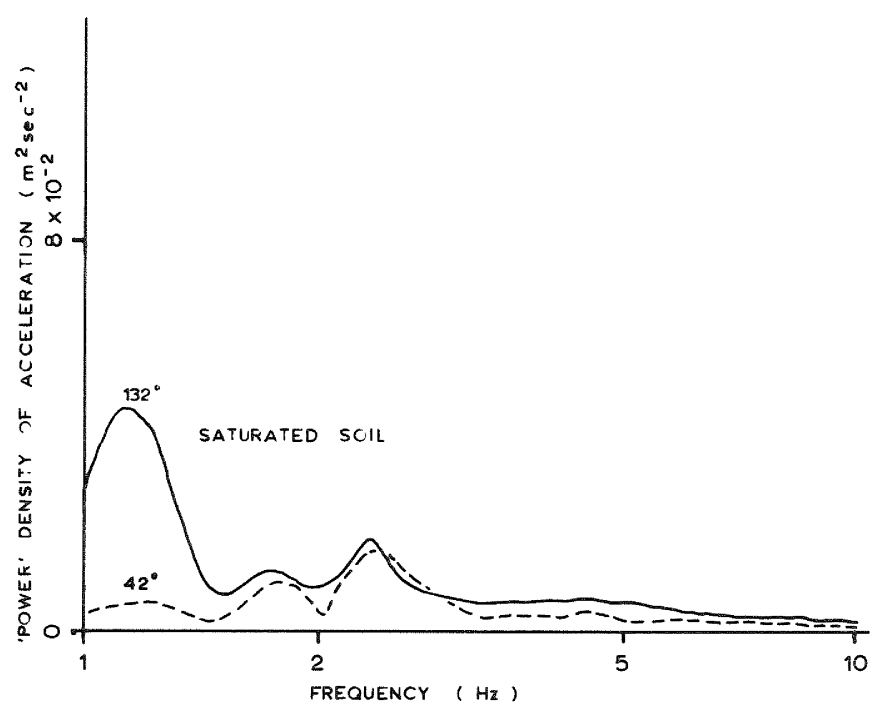

FIGURE 8: TE ARO POST OFFICE, $6 / 1 / 73$

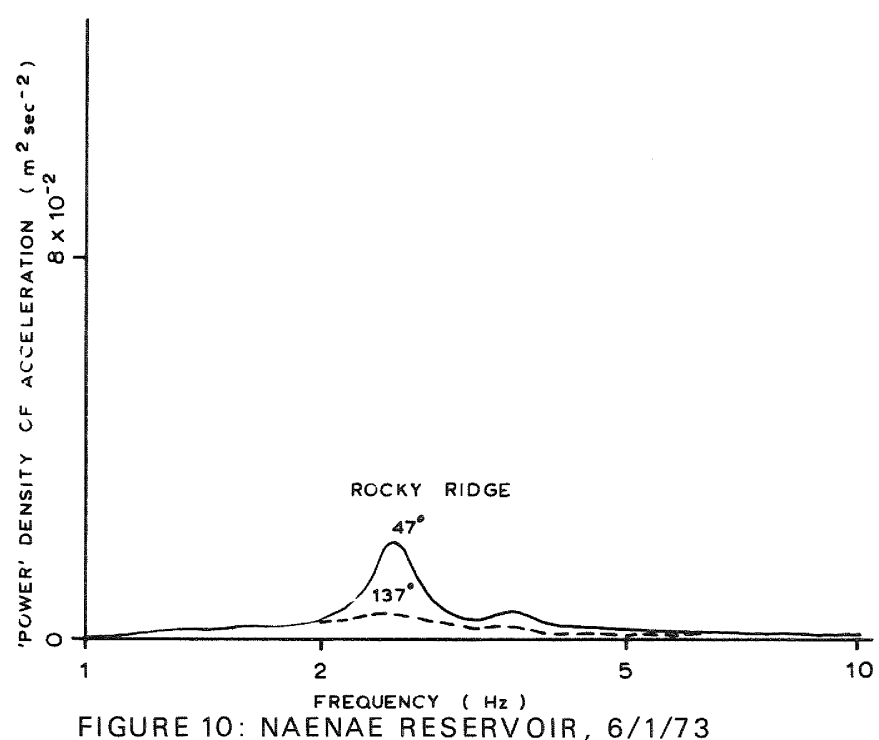




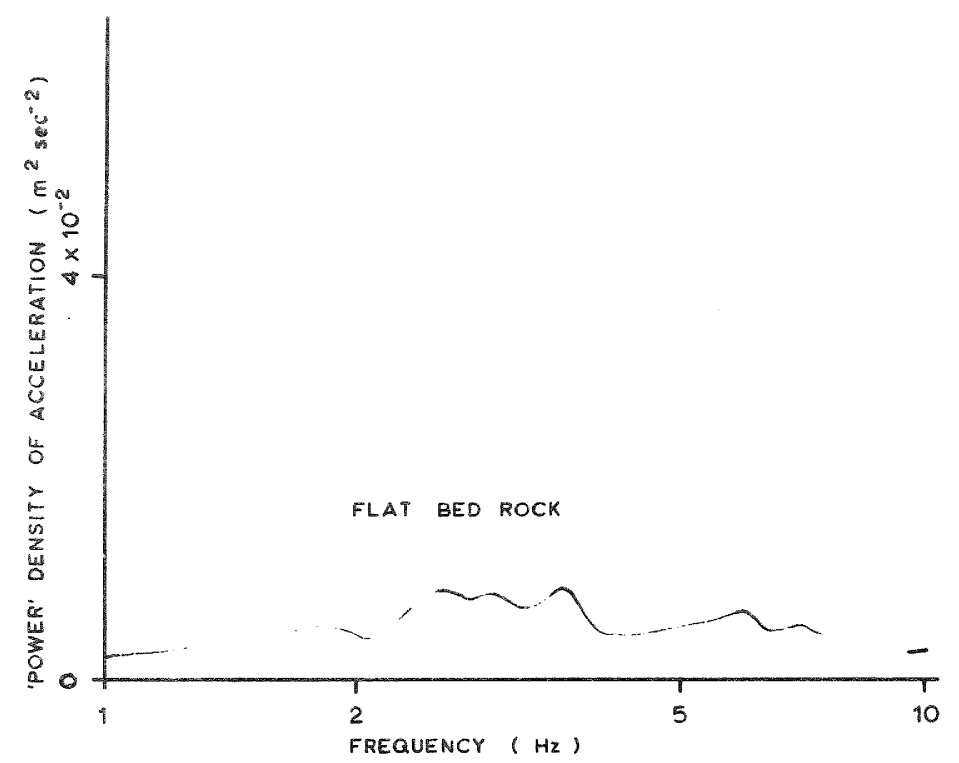

FIGURE 11: INSTITUTE OF NUCLEAR SCIENCES,

6/1/73 TOTAL POWER (AZIMUTH INDEPENDENT)

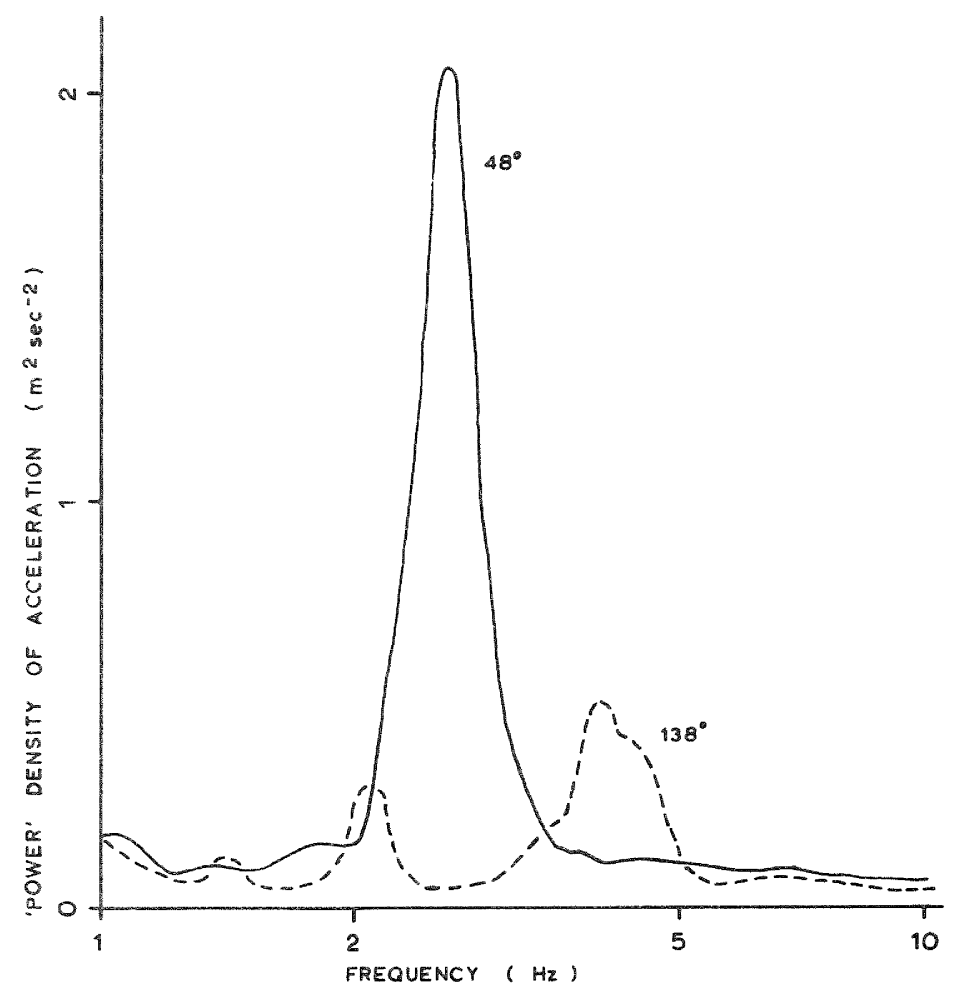

FIGURE 13: TEMBLOR, 27/6/66

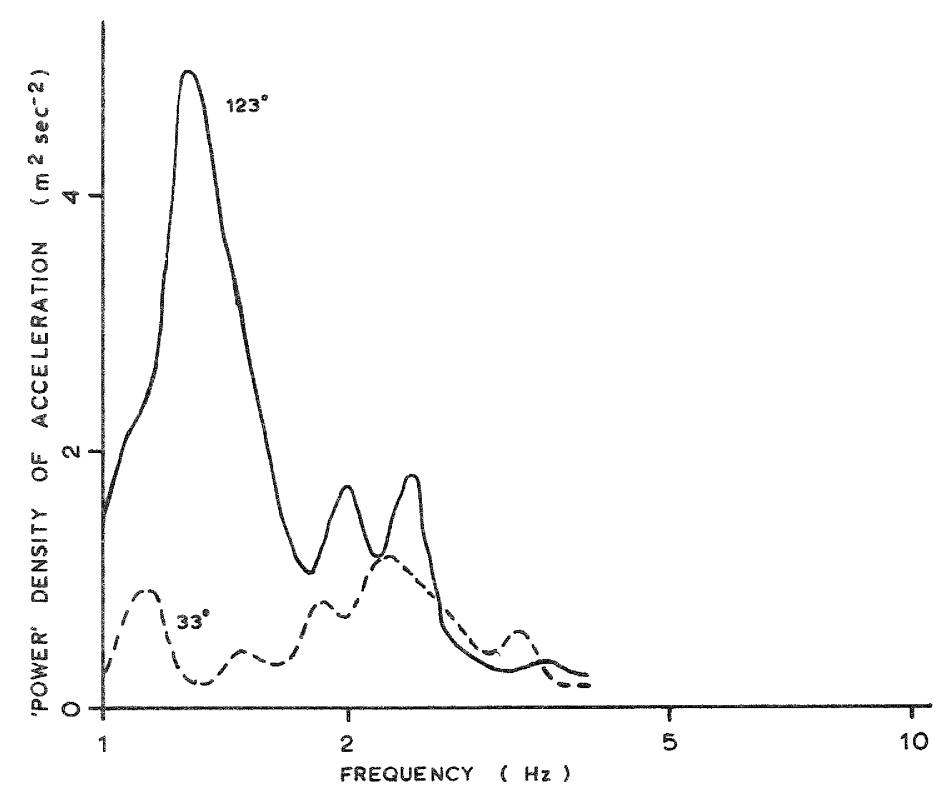

FIGURE 15: LEUKAS MAIN SHOCK, $4 / 11 / 73$

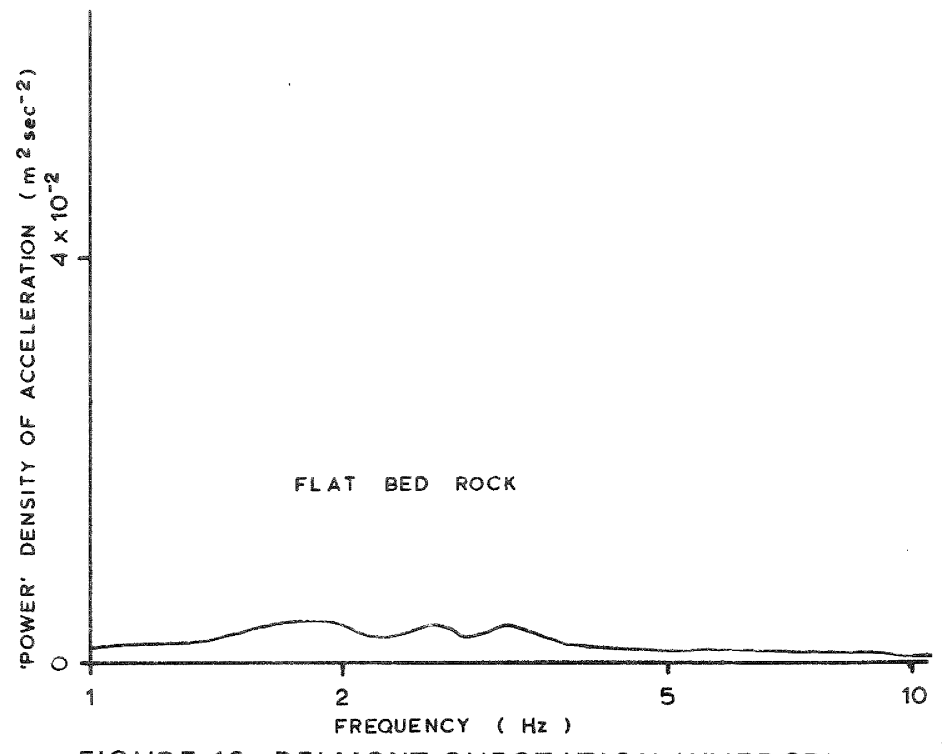

FIGURE 12: BELMONT SUBSTATION (HVEPGB), $6 / 1 / 73$ TOTAL POWER (AZIMUTH INDEPENDENT)

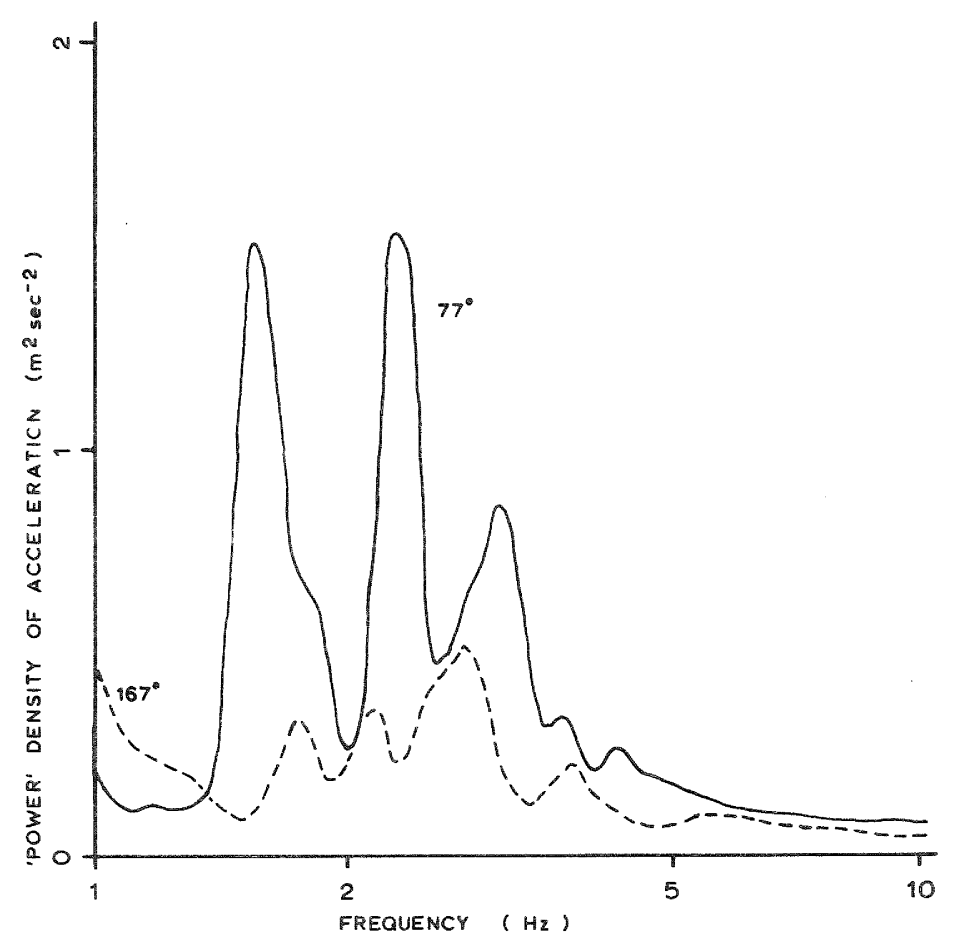

FIGURE 14: EUREKA, 21/12/54

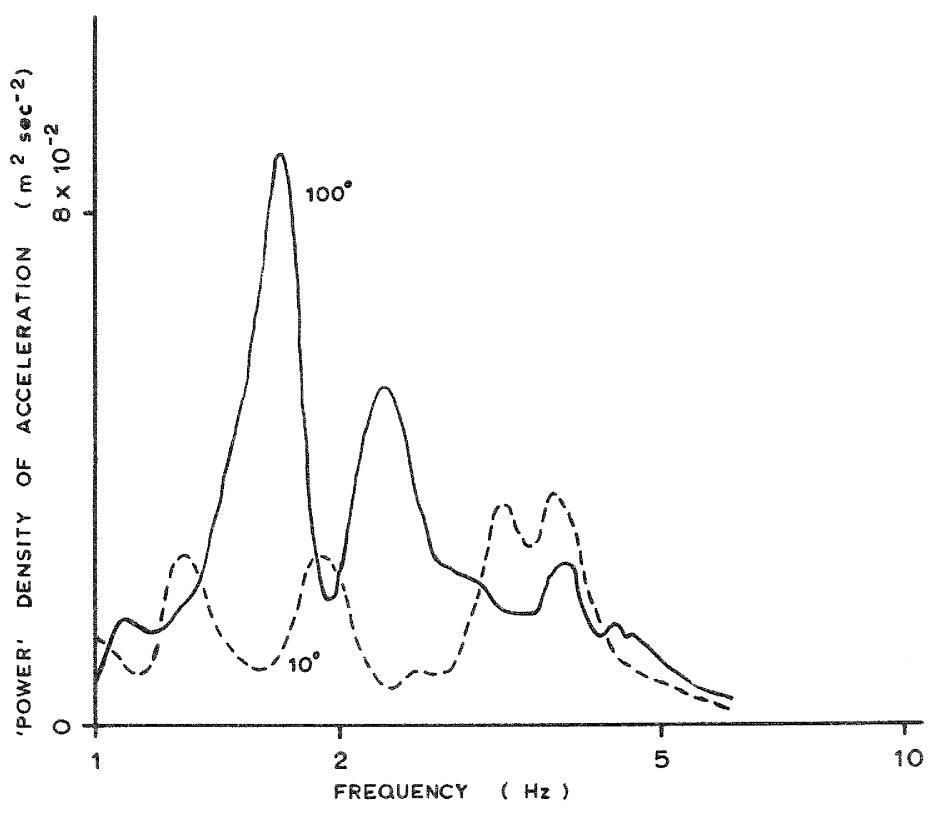

FIGURE 16: LEUKAS AFTERSHOCK, NOVEMBER 73 


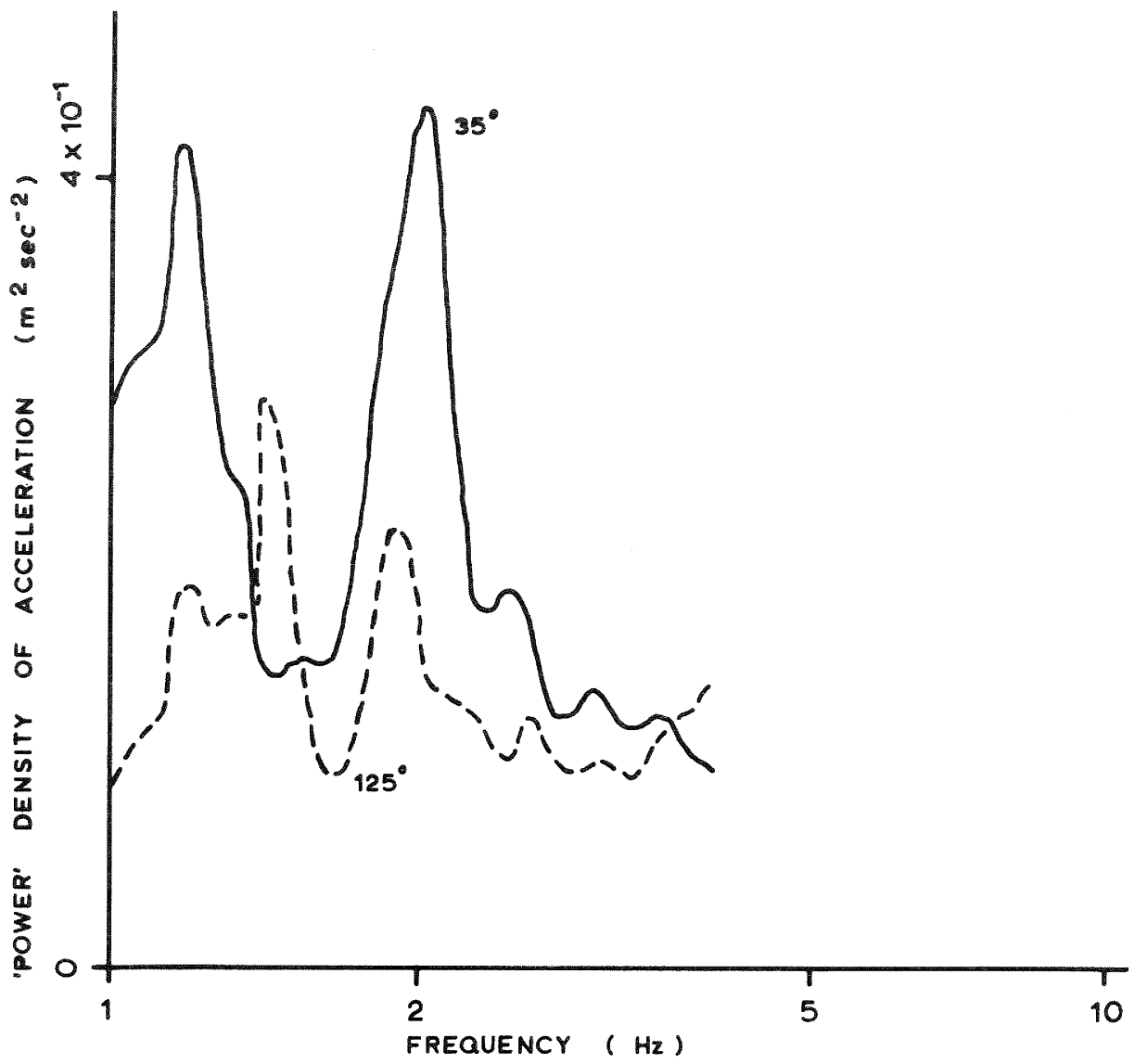

FIGURE 17: NIIGATA, 16/6/64

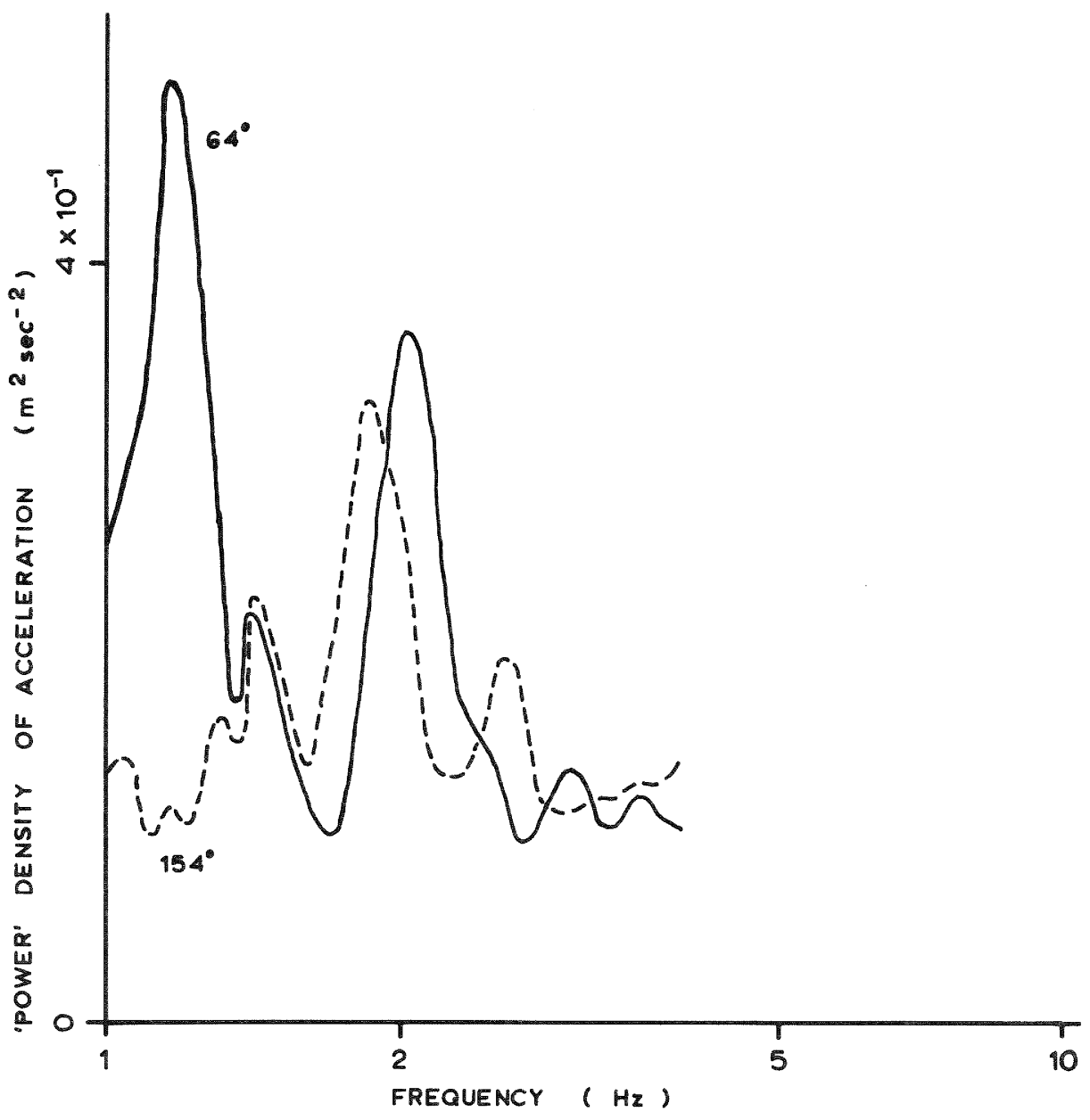

FIGURE 18: NIIGATA, 16/6/64 
FIGURE 19: CELLULAR MODE RESPONSE IN A SCALE MODEL

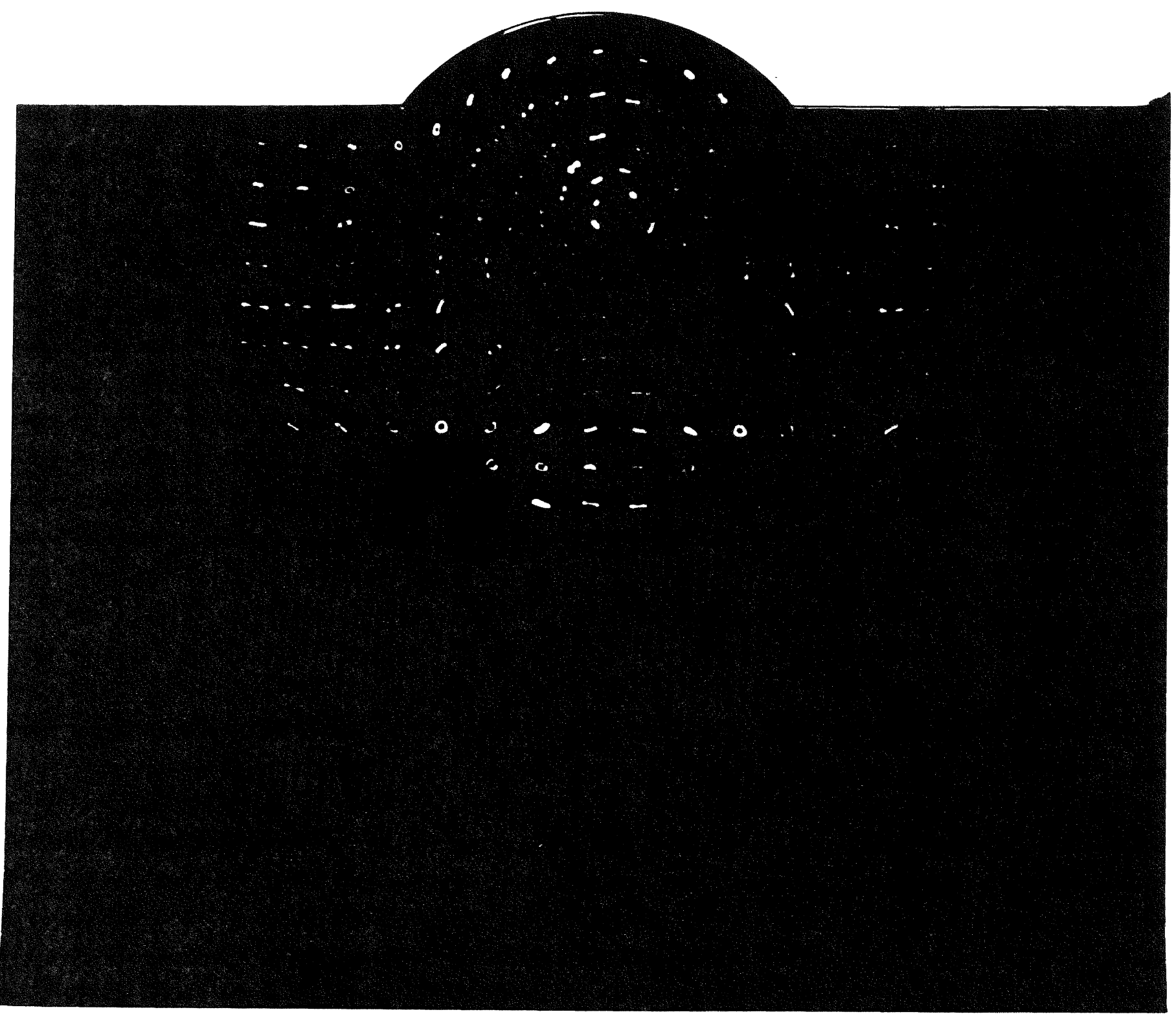

PLAN VIEW OF A RIGID CONTAINER FULL OF JELLY, SHAKEN SINUSOIDALLY IN A DIRECTION PARALLEL TO THE SIDE WITH AN INCLUDED CURVE. LIGHTWEIGHT MARKERS HAVE BEEN PLACED ON THE SURFACE, AND A TIME EXPOSED PHOTOGRAPH TAKEN. THE CLOSED PATHS SHOWN ARE CHARACTERISTIC OF CELLULAR MODE RESPONSE. THE PATTERN ONLY EXISTS WHEN THE MODEL IS EXCITED AT THE NATURAL FREQUENCY OF THE MODE. 